\title{
The evaluation of implant stability measured by resonance frequency analysis in different bone types
}

\author{
Naser Sargolzaie', Sarah Samizade ${ }^{2}$, Hamidreza Arab, \\ Habibollah Ghanbari', Leila Khodadadifard ${ }^{1}$, Amin Khajavi ${ }^{1, *}$ \\ ${ }^{I}$ Department of Periodontics, College of Dentistry, Mashhad University of Medical Science, Mashhad, \\ ${ }^{2}$ Department of Periodontics, College of Dentistry, Sabzevar University of Medical Science, Sabzevar, Iran
}

\begin{abstract}
J Korean Assoc Oral Maxillofac Surg 2019;45:29-33)
Objectives: Bone density seems to be an important factor affecting implant stability. The relationship between bone density and primary and secondary stability remains under debate. The aim of this study was to compare primary and secondary stability measured by resonance frequency analysis (RFA) between different bone types and to compare implant stability at different time points during 3 months of follow-up.

Materials and Methods: Our study included 65 implants (BioHorizons Implant Systems) with 3.8 or $4.6 \mathrm{~mm}$ diameter and 9 or $10.5 \mathrm{~mm}$ length in 59 patients. Bone quality was assessed by Lekholm-Zarb classification. After implant insertion, stability was measured by an Osstell device using RFA at three follow-up visits (immediately, 1 month, and 3 months after implant insertion). ANOVA test was used to compare primary and secondary stability between different bone types and between the three time points for each density type.

Results: There were 9 patients in type I, 18 patients in type II, 20 patients in type III, and 12 patients in type IV. Three implants failed, 1 in type I and 2 in type IV. Stability values decreased in the first month but increased during the following two months in all bone types. Statistical analysis showed no significant difference between RFA values of different bone types at each follow-up or between stability values of each bone type at different time points.

Conclusion: According to our results, implant stability was not affected by bone density. It is difficult to reach a certain conclusion about the effect of bone density on implant stability as stability is affected by numerous factors.
\end{abstract}

Key words: Dental implant, Implant stability, Implant stability quotient

[paper submitted 2018. 4. 12 / revised 2018. 5. 23 / accepted 2018. 5. 23]

\section{Introduction}

Dental implants are considered as a treatment for completely or partially edentulous patients, with a success rate of $98 \%{ }^{1}$. Successful treatment depends on patient-related parameters and the surgical procedure ${ }^{2}$. Preoperative assess-

\section{Leila Khodadadifard}

Department of Periodontics, College of Dentistry, Mashhad University of Medical Science, Park Square, Mashhad 9197995185, Iran

TEL: +98-9153057255 FAX: +98-5137669028

E-mail: l.khodadadifard@gmail.com

ORCID: https://orcid.org/0000-0002-7517-2152

*Current affiliation: Department of Periodontics, College of Dentistry, Bojnurd University of Medical Science, Bojnurd, Iran

(c) This is an open-access article distributed under the terms of the Creative Commons Attribution Non-Commercial License (http://creativecommons.org/ licenses/by-nc/4.0/), which permits unrestricted non-commercial use, distribution, and reproduction in any medium, provided the original work is properly cited.

Copyright (C) 2019 The Korean Association of Oral and Maxillofacial Surgeons. All rights reserved. ment of available bone and its quality affects the selection of surgical technique and implant site and design, which will improve the success rate of implantation ${ }^{3,4}$. Some authors have attributed the higher success of mandibular implants to its better bone quality ${ }^{5}$. Bone density relates to its biomechanical features, which depend on many other factors including mineralization and structure. Many classifications have been suggested for bone density ${ }^{6}$, and the most common one is a 4-type scale proposed by Lekholm-Zarb classification. This assortment is based on radiographic evaluation and bone resistance assessed by the surgeon while drilling ${ }^{7}$. Implant stability is affected by bone quality and quantity as well as implant design and operative technique ${ }^{2}$. The authors suggest that implant stability is one of the important prerequisites for osteointegration $^{8,9}$. Different methods have been proposed for evaluation of implant stability. Although percussion and radiographic assessment are commonly used, their results are not always reliable even in cases of fibrous encapsulation ${ }^{10}$. 
Periotest values and resonance frequency analysis (RFA) respectively measured by periotest and Osstell device are additional methods for evaluation of implant stability ${ }^{11}$.

Studies have shown that measurement of implant stability with RFA is reliable, noninvasive, and can be used at any time after implant insertion ${ }^{12}$. The aim of this study was to compare primary and secondary stability measured by RFA between different bone types and to compare implant stability at different time points during 3 months of follow-up.

\section{Materials and Methods}

\section{Patients}

This study enrolled 59 patients (30 men and 29 women) who were referred to the Department of Periodontics, College of Dentistry, Mashhad University of Medical Science (Mashhad, Iran) from April to June 2017 and who had a mean age of $54 \pm 12$ years. All patients signed informed consent before surgery. The protocol of the study and consent were confirmed by the ethics committee of Mashhad University of Medical Sciences (approval no. IR.mums.sd.REC.1394.158). Patients with uncontrolled systemic diseases, immunodeficiency, who were contraindicated for implant surgery and cone-beam computed tomography (CBCT) imaging, and who needed bone graft before implant placement were excluded from the study. CBCT imaging was done for all subjects; using these images, they were classified into 4 types based on Lekholm-Zarb classification: type I, compact homogenous bone; type II, thich layer of cortical bone encircles a core of dens trabecular bone; type III, thin layer of cortical bone encircles a core of dens trabecular bone; and type IV, very thin layer of cortical bone encircles a core of trabecular bone with low density. Type I was seen in 9 patients, type II in 18 patients, type III in 20 patients and type IV in 12 patients.

\section{Surgical procedure}

All the patients were premedicated with chlorohexidine $0.2 \%$ mouth rinse before surgery. After local anesthesia, crestal incision was performed, and the full periosteal flap was elevated. The 65 tapered bone level implants (BioHorizons Implant Systems, Brimingham, AL, USA) had 3.8 or $4.6 \mathrm{~mm}$ diameter and 9 or $10.5 \mathrm{~mm}$ length. The healing abutment was placed, and the flap was tied with 4-0 silk suture. All procedures were performed by the same surgeon with a certain non-submerged protocol. Amoxicillin $500 \mathrm{mg}$ (three times a day, for 7 days), gelofen (400 mg, four times a day, for 7 days, in case of pain) and chlorohexidine $0.2 \%$ mouth wash were prescribed.

\section{Resonance frequency analysis}

RFA was measured by an Osstell instrument (Integration Diagnostics, Göteborg, Sweden) at baseline (immediately after insertion) and 1 and 3 months after surgery.(Fig. 1) For this measurement, a transducer with $8.5 \mathrm{~mm}$ length was placed on the fixtures. The resonance frequency (RF) transducer consisted of two piezoceramic elements attached to an offset cantilever beam. Stimulation of the elements causes vibration of the beam. The stimulating signal is a sinusoid wave with frequency of 5 to $15 \mathrm{~Hz}$ and amplitude peak of $1 \mathrm{~V}$. $\mathrm{RF}$ values are recorded as implant stability quotient (ISQ) on a scale from 1 to 100 .

\section{Statistical analysis}

ANOVA and Kruskal-Wallis test were used for comparison of ISQ between different bone densities at each time point and also between density types at different time points. Kolmogrov-Smirnov test for normal distribution and post hoc (Tukey) test for pair-comparison were used. Statistical analysis was performed using SPSS (ver. 16; SPSS Inc., Chicago, IL, USA) and $P<0.05$ was considered significant.

\section{Results}

This study enrolled 59 patients (29 women and 30 men)

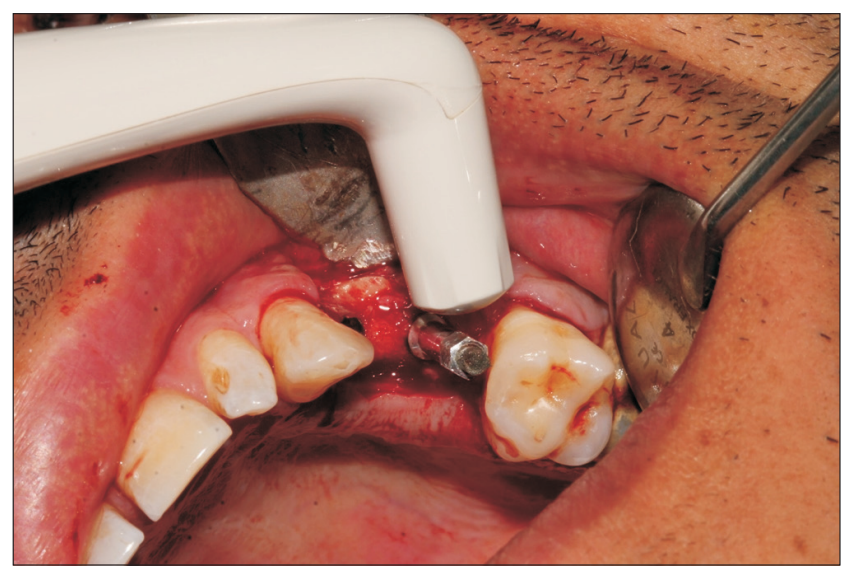

Fig. 1. Measurment of implant stability by an Osstell device (Integration Diagnostics) immediately after installation.

Naser Sargolzaie et al: The evaluation of implant stability measured by resonance frequency analysis in different bone types. J Korean Assoc Oral Maxillofac Surg 2019 
Table 1. Implant stability quotient (ISQ) immediately after insertion

\begin{tabular}{lc}
\hline Bone type & ISQ value \\
\hline I & $77.21 \pm 4.25$ \\
II & $74.40 \pm 2.12$ \\
III & $76.61 \pm 3.45$ \\
IV & $73.50 \pm 4.43$ \\
$P$-value & 0.124 \\
\hline
\end{tabular}

Values are presented as mean \pm standard deviation.

Lekholm-Zarb classification: type I, compact homogenous bone; type II, thich layer of cortical bone encircles a core of dens trabecular bone; type III, thin layer of cortical bone encircles a core of dens trabecular bone; and type IV, very thin layer of cortical bone encircles a core of trabecular bone with low density.

Naser Sargolzaie et al: The evaluation of implant stability measured by resonance frequency analysis in different bone types. J Korean Assoc Oral Maxillofac Surg 2019

Table 2. Implant stability quotient (ISQ) one month after insertion

\begin{tabular}{cc}
\hline Bone type & ISQ value \\
\hline I & $68.60 \pm 2.13$ \\
II & $70.50 \pm 2.78$ \\
III & $72.74 \pm 2.45$ \\
IV & $69.13 \pm 2.65$ \\
$P$-value & 0.431 \\
\hline
\end{tabular}

Values are presented as mean \pm standard deviation.

Refer to Table 1 for the definition of bone types.

Naser Sargolzaie et al: The evaluation of implant stability measured by resonance frequency analysis in different bone types. J Korean Assoc Oral Maxillofac Surg 2019

with an age range of 35 to 78 years (mean, $54 \pm 12$ years). In these patients, 65 implants were inserted in different regions. According to Lekholm-Zarb classification, patients were categorized into 4 types: Types I to IV had 9, 18, 20, and 12 patients, respectively. In our study, 3 implants failed (1 in type I and 2 in type IV). At implant installation, the mean ISQ value was $77.21 \pm 4.25$ in type I, $74.40 \pm 2.12$ in type II, $76.61 \pm 3.45$ in type III, and $73.50 \pm 4.43$ in type IV.(Table 1) One month after implant insertion, the mean ISQ values were $68.60 \pm 2.13,70.50 \pm 2.78,72.74 \pm 2.45$, and $69.13 \pm 2.65$ in types I to IV, respectively.(Table 2) The mean ISQ values at the 3-month follow-up were 74.23 \pm 2.54 in type I, 76.10 \pm 3.23 in type II, 78.70 \pm 3.75 in type III, and $73.46 \pm 3.32$ in type IV.(Table 3) According to statistical analysis, these values were not significantly different at any time point $(P>0.05)$. The implant stability of each bone type at baseline, 1 month, and 3 months after insertion were compared and showed no statistically significant difference $(P>0.05)$.(Fig. 2$)$

\section{Discussion}

Implant stability is one of the most important factors for successful implant treatment and seems to be crucial for osteointegration, especially for immediate loading. Primary sta-
Table 3. Implant stability quotient (ISQ) three months after insertion

\begin{tabular}{lc}
\hline Bone type & ISQ value \\
\hline I & $74.23 \pm 2.54$ \\
II & $76.10 \pm 3.23$ \\
III & $78.70 \pm 3.75$ \\
IV & $73.46 \pm 3.32$ \\
$P$-value & 0.094 \\
\hline
\end{tabular}

Values are presented as mean \pm standard deviation.

Refer to Table 1 for the definition of bone types.

Naser Sargolzaie et al: The evaluation of implant stability measured by resonance frequency analysis in different bone types. J Korean Assoc Oral Maxillofac Surg 2019

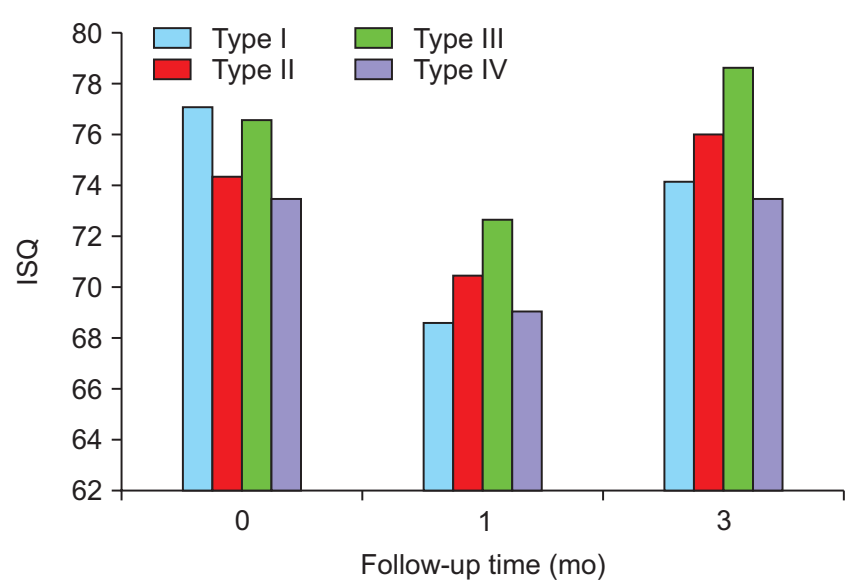

Fig. 2. Mean implant stability quotient (ISQ) values of buccolingual measurements by an Osstell instrument (Integration Diagnostics) in each bone type at different follow-up times. Refer to Table 1 for the definition of bone types.

Naser Sargolzaie et al: The evaluation of implant stability measured by resonance frequency analysis in different bone types. J Korean Assoc Oral Maxillofac Surg 2019

bility is a mechanical phenomenon, while secondary stability is the result of a biological event (osteointegration) ${ }^{13}$. Studies have shown that factors including bone density, implant size and design, and operative technique may influence implant primary stability ${ }^{14}$. On the other hand, secondary stability depends on ossification around the implant fixture ${ }^{15}$. According to our results, for all bone types, stability values decreased during the first month and increased in the following two months. However, these changes were not statistically significant.

Many authors have described changes of implant stability over time. Most of these studies showed a similar pattern of consecutive reduction and increase in ISQ value. There remains debate on the timing of stability changes. Studies have reported an ISQ reduction period from the 1st to 8th week after implant installation ${ }^{16-18}$. According to Simunek et al. ${ }^{17}$, only implants with low primary stability showed increase of ISQ during the healing period, while implants with high pri- 
mary stability showed reduction of stability values. Martinez et al. ${ }^{19}$ showed that primary stability was different among various bone densities, but secondary stability was similar. Farré-Pagés et al. ${ }^{1}$ found a significant relationship between bone density according to Lekholm-Zarb classification and ISQ value; as a result, implant stability in type I was significantly higher than other types. Turkyilmaz et al. ${ }^{20}$, Ikumi and Tsutsumi ${ }^{21}$, and Friberg et al. ${ }^{22}$ showed a significant relationship between bone density and primary and secondary stability. The results of studies have not been consistent about the relationship among bone density and primary and secondary stability. For example, in a study by Beer et al. ${ }^{2}$, there was no statistically significant relationship between bone density and primary stability. In another study, Simunek et al. ${ }^{17}$ could not show a significant relationship between bone type and primary stability or primary and secondary stability. Similarly, in the present study, primary and secondary stability were not significantly difference among various bone types. A recent review article reported that results on the relationship between bone density and implant stability were not consistent, and this controversy could be attributed to diverse methodology of studies, different descriptions and evaluation of bone quality, and different methods of stability assessment ${ }^{23}$. Various implant sizes and designs could be another source of difference, as implants with greater diameter have higher primary stability due to additional bone-to-implant contact (BIC). Also, tapered implants have higher primary stability because of greater diameter at the crestal region ${ }^{19}$. Implant surface modification is another possible source of difference and may have an effect on osteointegration and $\mathrm{BIC}^{24}$. Although Han et al. ${ }^{18}$ did not find a relationship of implant surface modification and diameter with ISQ value. Regardless of the relationship between density and primary and secondary stability, it seems that implants with primary stability lower than a critical limit cannot achieve secondary stability. Different critical limits have been proposed, although ISQ $<50$ is considered an acceptable limit for primary stability, and successful integration could be possible in this group. Implants with ISQ $>60$ had more chance of successful osteointegration ${ }^{25,26}$. In our study, failure was seen in all three implants with primary stability of ISQ $<50$.

\section{Conclusion}

According to our results, stability decreased during the first month and increased in the following two months for all bone types. These changes were not statistically significant.
The ISQ values of all bone types were not statistically significant different at each time point. Studies have not shown consistent results on the relationship between bone density and implant stability. Due to various methodologies for stability measurement, density classification, different follow-up times, and diverse implant surface and design, it is very challenging to reach a conclusion about the importance of stability in osteointegration. The effects of other factors, especially implant surface and design and surgical technique, on the relationship between primary stability and osteointegration should be evaluated in future studies.

\section{ORCID}

Naser Sargolzaie, https://orcid.org/0000-0002-7183-9191

Sarah Samizade, https://orcid.org/0000-0002-4492-0031

Hamidreza Arab, https://orcid.org/0000-0003-0910-3201

Habibollah Ghanbari, https://orcid.org/0000-0003-44732505

Leila Khodadadifard, https://orcid.org/0000-0002-75172152

Amin Khajavi, https://orcid.org/0000-0003-3916-3088

\section{Authors' Contributions}

S.S. and H.A. participated in data collection. L.K. wrote the manuscript and participated in the study design. N.S. and H.G. participated in the study design and coordination. A.K. performed the statistical analysis and helped to draft the manuscript. All authors read and approved the final manuscript.

\section{Ethics Approval and Consent to Participate}

The protocol of the study and consent were confirmed by the ethics committee of Mashhad University of Medical Sciences (approval no. IR.mums.sd.REC.1394.158). All patients signed informed consent before surgery.

\section{Conflict of Interest}

No potential conflict of interest relevant to this article was reported.

\section{References}

1. Farré-Pagés N, Augé-Castro ML, Alaejos-Algarra F, MarequeBueno J, Ferrés-Padró E, Hernández-Alfaro F. Relation between bone density and primary implant stability. Med Oral Patol Oral 
Cir Bucal 2011;16:e62-7.

2. Beer A, Gahleitner A, Holm A, Tschabitscher M, Homolka P. Correlation of insertion torques with bone mineral density from dental quantitative $\mathrm{CT}$ in the mandible. Clin Oral Implants Res 2003;14:616-20.

3. Ekfeldt A, Christiansson U, Eriksson T, Lindén U, Lundqvist S, Rundcrantz T, et al. A retrospective analysis of factors associated with multiple implant failures in maxillae. Clin Oral Implants Res 2001;12:462-7.

4. Park SW, Jang SM, Choi BH, Son HN, Park BC, Kim CH, et al. The study of bone density assessment on dental implant sites. J Korean Assoc Oral Maxillofac Surg 2010;36:417-22.

5. Turkyilmaz I, Tözüm TF, Tumer C. Bone density assessments of oral implant sites using computerized tomography. J Oral Rehabil 2007;34:267-72.

6. Ribeiro-Rotta RF, Lindh C, Pereira AC, Rohlin M. Ambiguity in bone tissue characteristics as presented in studies on dental implant planning and placement: a systematic review. Clin Oral Implants Res 2011;22:789-801.

7. Turkyilmaz I, McGlumphy EA. Influence of bone density on implant stability parameters and implant success: a retrospective clinical study. BMC Oral Health 2008;8:32.

8. Albrektsson T, Brånemark PI, Hansson HA, Lindström J. Osseointegrated titanium implants. Requirements for ensuring a longlasting, direct bone-to-implant anchorage in man. Acta Orthop Scand 1981;52:155-70.

9. Szmukler-Moncler S, Salama H, Reingewirtz Y, Dubruille JH. Timing of loading and effect of micromotion on bone-dental implant interface: review of experimental literature. J Biomed Mater Res 1998;43:192-203.

10. Makary C, Rebaudi A, Sammartino G, Naaman N. Implant primary stability determined by resonance frequency analysis: correlation with insertion torque, histologic bone volume, and torsional stability at 6 weeks. Implant Dent 2012;21:474-80.

11. Coutant JC, Seguela V, Hauret L, Caix P, Ella B. Assessment of the correlation between implant stability and bone density by computed tomography and resonance frequency analysis in fresh cadavers. Int J Oral Maxillofac Implants 2014;29:1264-70.

12. Kahraman S, Bal BT, Asar NV, Turkyilmaz I, Tözüm TF. Clinical study on the insertion torque and wireless resonance frequency analysis in the assessment of torque capacity and stability of selftapping dental implants. J Oral Rehabil 2009;36:755-61.

13. Atsumi M, Park SH, Wang HL. Methods used to assess implant stability: current status. Int J Oral Maxillofac Implants 2007;22:74354.

14. Sennerby L, Meredith N. Implant stability measurements using resonance frequency analysis: biological and biomechanical aspects and clinical implications. Periodontol 2000 2008;47:51-66.

15. Meredith N. Assessment of implant stability as a prognostic determinant. Int J Prosthodont 1998;11:491-501.

16. Kavita GP, Bansi MB, Rizwan MS. Clinical evaluation of primary stability and osseointegration of endosseous root form implants of different dimensions using periotest. Indian J Appl Res 2013;3:2813.

17. Simunek A, Kopecka D, Brazda T, Strnad I, Capek L, Slezak R. Development of implant stability during early healing of immediately loaded implants. Int J Oral Maxillofac Implants 2012;27:61927.

18. Han J, Lulic M, Lang NP. Factors influencing resonance frequency analysis assessed by Osstell mentor during implant tissue integration: II. Implant surface modifications and implant diameter. Clin Oral Implants Res 2010;21:605-11.

19. Martinez H, Davarpanah M, Missika P, Celletti R, Lazzara R. Optimal implant stabilization in low density bone. Clin Oral Implants Res 2001;12:423-32.

20. Turkyilmaz I, Tözüm TF, Tumer C, Ozbek EN. Assessment of correlation between computerized tomography values of the bone, and maximum torque and resonance frequency values at dental implant placement. J Oral Rehabil 2006;33:881-8.

21. Ikumi N, Tsutsumi S. Assessment of correlation between computerized tomography values of the bone and cutting torque values at implant placement: a clinical study. Int J Oral Maxillofac Implants 2005;20:253-60

22. Friberg B, Jisander S, Widmark G, Lundgren A, Ivanoff CJ, Sennerby L, et al. One-year prospective three-center study comparing the outcome of a "soft bone implant" (prototype Mk IV) and the standard Brånemark implant. Clin Implant Dent Relat Res 2003;5:717.

23. Miri R, Shirzadeh A, Kermani H, Khajavi A. Relationship and changes of primary and secondary stability in dental implants: a review. Int J Contemp Dent Med Rev 2017;2017:03011.

24. Geckili O, Bilhan H, Bilgin T. A 24-week prospective study comparing the stability of titanium dioxide grit-blasted dental implants with and without fluoride treatment. Int J Oral Maxillofac Implants 2009;24:684-8.

25. Zhou W, Han C, Yunming L, Li D, Song Y, Zhao Y. Is the osseointegration of immediately and delayed loaded implants the same?: comparison of the implant stability during a 3-month healing period in a prospective study. Clin Oral Implants Res 2009;20:13606.

26. Degidi M, Daprile G, Piattelli A. Determination of primary stability: a comparison of the surgeon's perception and objective measurements. Int J Oral Maxillofac Implants 2010;25:558-61. 